\title{
Serge Valletti : portrait de l'artiste en Don Quichotte
}

\section{Mireille Losco-Lena}

\section{(2) OpenEdition}

1 Journals

Édition électronique

URL : http://journals.openedition.org/recherchestravaux/151

DOI : 10.4000/recherchestravaux.151

ISSN : 1969-6434

Éditeur

UGA Éditions/Université Grenoble Alpes

Édition imprimée

Date de publication : 15 octobre 2006

Pagination : 17-25

ISBN : 978-2-84310-099-4

ISSN : 0151-1874

\section{Référence électronique}

Mireille Losco-Lena, "Serge Valletti : portrait de l'artiste en Don Quichotte », Recherches \& Travaux [En ligne], 69 | 2006, mis en ligne le 13 mars 2009, consulté le 07 septembre 2020. URL : http://

journals.openedition.org/recherchestravaux/151; DOI : https://doi.org/10.4000/recherchestravaux. 151 


\section{Serge Valletti : portrait de l'artiste en Don Quichotte}

Je me demande [...] si je ne vais pas arrêter tout simplement de faire quoi que ce soit et si je ne vais pas relire indéfiniment le même livre... L'histoire scandaleuse de Don Quichotte de la Manche et de son acolyte Sancho Pança. Que je ferais jouer par des femmes-marionnettes qui n'arrêteraient pas de se demander ce qu'elles sont en train de faire.

Ainsi s'explique le personnage de l'Introduction destinée à faire comprendre dans quel état je suis lorsque j'envisage de me mettre au travail en vue de fabriquer un spectacle seul, solo de Serge Valletti introduisant à cinq autres solos écrits et joués par l'auteur dans les années I980․ Face à l'angoisse de la page blanche et des yeux du public tapi dans le noir, angoisse qui est au centre de cette pièce, Don Quichotte semble venir à la rescousse pour délier la parole du dramaturge et autoriser l'acteur à toutes les fantaisies. Serge Valletti, auteur et acteur, serait peut-être ce «Don Quichotte du dimanche» dont il est question dans $A u$ bout du comptoir, la mer ${ }^{2}$; toute son œuvre viendrait se fondre dans l'ouvrage de Cervantès ou bien la rejouer sous les formes les plus drolatiques qui soient. À y bien réfléchir, il est logique que Don Quichotte, qui est l'un des plus grands mythomanes de l'imaginaire occidental, qui en constitue même le modèle le plus piteusement héroïque, apparaisse comme la figure tutélaire de tous les personnages mythomanes de Valletti. Ce dernier ne cache pas la sympathie profonde qu'il leur voue: "moi metteur en scène de théâtre, qui ne suis pas mythomane mais qui par contre ai beaucoup d'amis mythomanes ${ }^{3}$ ». Dans une

* Avec d'amicaux remerciements à Dominique Laidet, qui m’a largement aidée à entrer dans l'œuvre de Serge Valletti.

I. Six Solos, Nantes, L'Atalante, 2004, p. 18.

2. Au bout du comptoir, la mer, dans Six Solos, ibid., p. 167.

3. L'Introduction..., dans Six Solos, ibid., p. 23. 
note de bas de page à la fin de l' Introduction..., l'auteur met en place une fiction selon laquelle les cinq solos publiés à la suite seraient des retranscriptions de la parole de ces amis mythomanes qu'il aurait rencontrés en prison. Ce serait donc à partir de l'expérience de l'enfermement, de l'incarcération - ou de la pauvreté du réel pour Don Quichotte - que l'affabulation mythomane se serait irrésistiblement déclenchée, ouvrant les portes de l'aventure, de l'errance et de la liberté. Sous le signe de l'épopée de Don Quichotte, les méandres de l'écriture de Valletti se vouent à l'exaltation du cheminement erratique, du mouvement à la dérive; il s'y exprime un désir irrépressible d'arpenter le monde au gré de l'aventure. Du reste, le terme d'aventure ne nous vient-il pas des épopées médiévales, de ces récits de chevaliers errants dont la lecture fascine tant Don Quichotte? C'est de la lecture des romans de chevalerie que part toute l'errance bouffonne du personnage. Or l'aventure, c'est le triomphe de ce qui advient; étymologiquement, le terme désigne cette idée du hasard qui hante l'écriture de Valletti et dont les personnages font parfois l'éloge. Ainsi dans Plus d'histoires, l'auteur met en scène un personnage débordé par des liasses de textes qui l'entraînent dans une aventure chaotique:

[...] ça le jeu du hasard, bon, ben autant le dire tout de suite c'est un peu la seule chose que je maîtrise. / Parce qu'au moins le hasard, c'est la seule chose qui ne trompe pas son monde! / Vous comprenez, on sait à quoi on s'attend avec le hasard, parce que, en admettant qu'il vous déçoive le hasard, eh bien, ce n'est pas une véritable déception, puisque c'était le hasard, donc il avait en quelque sorte prévenu, le hasard. / C'est ça qui est bien dans lui! / Et quand il vous fait une bonne surprise, eh bien, on sait la saluer avec tous les honneurs qui lui sont dûs, au hasard! ${ }^{4}$

En vertu du règne du hasard, l'aventure à la dérive des personnages de Valletti devient une chanson de geste profondément jubilatoire, suscitant le rire mais aussi le décentrement de notre regard sur le monde et la réévaluation positive des illusions ou du jeu avec l'imaginaire.

\section{Un comique de la jubilation}

Le comique chez Valletti a fondamentalement partie liée avec la jubilation, une jubilation que le spectateur partage avec l'auteur-acteur. Ce dernier est en effet le premier à jubiler en scène: «c'est ma principale activité d'organiser le rire. C'est ça, j'organise le rire. Et moi je ris de mon propre rires». Ce rire exponentiel, qui se multiplie de se regarder lui-même, renvoie au mouvement d'expansion propre à la jubilation. Car si la jubilation accompagne souvent

4. Plus d'histoires, prologue pour un nouveau théâtre, dans La Revue du théâtre, 1994, p. 38.

5. Abécédaire, "I comme Hilare», site internet officiel de l'auteur, http://perso.wanadoo. $\mathrm{fr} /$ serge.valletti/ 
l'effet comique, elle ne lui est pas intrinsèquement liée. Il faut, pour que le rire devienne jubilatoire, un effet de propagation qui exige une durée et un espace démultipliés. On ne jubile pas d'un effet comique isolé, ponctuel, mais d'un mouvement d'accumulation, ou plutôt de prolifération infinie. Il faut l'étirement délirant des aventures de Don Quichotte, l'accumulation vertigineuse des chapitres, pour que l'aventure atteigne son point le plus irrésistible. Le comique jubilatoire se développe ainsi dans le temps, et a donc largement à voir avec l'aventure du spectacle dans son ensemble, depuis son début jusqu’à sa fin. Ce comique de mouvement naît des aléas, bonheurs et malheurs, trouvailles et déceptions, réussites et échecs de l'aventure. Il surgit de l'accumulation, au cours du spectacle, de ces aléas, de cette multitude de hasards qui nous donnent à jouir du désordre — nous ramenant sans doute à cet état d'enfance que nous portons tous en nous, d'où l'on tire sa joie de la défaite de l'ordre, du chaos galopant, voire de la destruction. Serge Valletti affectionne tout particulièrement les mots qui renvoient à ce bonheur du désordre: dans Plus d'histoires, le personnage est «emberlificoté au milieu de quelques dizaines de liasses de papier scribouillé»; dans Le jour se lève, Léopold!, il est question d' "amphigouri", de "cirque", de "tintamarre"; toujours dans l'ordre du sonore, on trouve dans Just Hamlet " un raffut de tous les diables»; dans Si vous êtes des hommes!, l'auteur nous parle de «bordel", de "souk». $\mathrm{Si}$, comme le dit le personnage de Balle perdue, "tellement de choses [...] se bousculent au portillon!», ou que, comme le dit cette fois-ci le personnage de $A$ u bout du comptoir la mer, "Je suis une casserole de lait qui déborde", c'est que pour Valletti il est toujours question de dérouler dans ses aventures "tout un attirail qui s'appelle le théâtre ${ }^{6}$. On ne pourra pas clore ce catalogue (qui est évidemment très loin d'être exhaustif!) sans faire référence au terme d'«embrouillamini», que l'auteur fait apparaître dans son Abécédaire pour illustrer la lettre L — allez savoir pourquoi!

C'est vrai que ça vient aussi un peu de ma manière d'écrire qui est de partir sans savoir où je vais et puis de trouver à la confusion un ordre et c'est comme ça que ça se passe $[\ldots]$ au départ accepter que ça puisse partir dans tous les sens, donc embrouillamini, oui...7

Même si du chaos des textes doit surgir un chemin, la part du désordre demeure assez puissante pour que scène et salle accèdent à la jubilation commune: «c'est

6. Plus d'histoires..., op. cit., p. 33; Le jour se lève, Léopold!, suivi de Souvenirs assassins, Paris, Christian Bourgois, 1988, p. 29, 73 et 87; Just Hamlet, dans Cinq Duos, Nantes, L'Atalante, 2004, p. 70 ; Si vous êtes des hommes! suivi de Réception, Nantes, L'Atalante, 1998, p. 50; Balle perdue, dans Six Solos, op. cit, p. 44; Au bout du comptoir, la mer, dans Six Solos, op. cit., p. 169; Introduction..., op. cit., p. 17 .

7. Abécédaire, op. cit., p. 7. 
l'avantage du théâtre, c'est que les gens qui vont au théâtre, ils s'installent dans des fauteuils et puis le rideau s'ouvre puis ils sont prêts à tout ${ }^{8} »$. Le chemin chez Valletti ne se construit jamais a priori, mais il se trouve à l'intérieur des méandres et des incongruités de l'aventure. La jubilation comique se fonde ainsi sur un émiettement de la représentation qui se refuse à faire synthèse.

Cette drôlerie repose sur une vision entropique du monde, c'est-à-dire sur une vision qui met l'accent sur l'accroissement du désordre, à l'exact inverse du travail d'apprentissage de la rigueur et de la structure auquel on soumet les enfants. Chez Valletti, la posture de gosse rétif à l'ordre semble rejoindre une métaphysique du chaos; le désordre du quotidien s'érige en vision du monde. Ainsi, tout mouvement est facteur de complexification, et faire le ménage devient une affaire impossible pour les personnages: le thème apparaît à la fois dans le premier acte de Le jour se lève, Léopold! où la scène encombrée embarrasse les personnages, et dans Souvenirs assassins:

Vous ne pouvez pas garder en mémoire tous vos souvenirs, il faut bien un peu faire du ménage de temps en temps. À force si vous ne rangez rien. Tout se mélange, s'emmêle et vous ne comprenez plus qui vous êtes!

On pourrait aussi parler de l'acte III de Si vous êtes des hommes!, où les personnages ont investi le musée de l'Homme et y sèment une pagaille irrépressible. Le mouvement entropique sera irréversible (ce qui est dans sa nature, bien sûr), le chaos menant les personnages à la mort finale. Mais là encore, on a des morts en série, comme si jusqu'au bout il fallait que cet univers prolifere!

Cette joie du désordre, même si elle contient sa part d'angoisse parce qu'elle touche au motif tragique de la catastrophe, ne peut jaillir que si le spectateur s'abandonne à la volupté du mouvement entropique. Il est invité à renoncer au désir de la maîtrise du sens et du mouvement. D'ailleurs «si la fin est au début et le début au milieu, ça n'a aucune importance ${ }^{\mathrm{I0}}{ }^{\prime}$. Maniaque de l'ordre s'abstenir! Le comique vallettien est aux antipodes de l'esthétique du ridicule et du comique de supériorité, où le rire sanctionne les errements des personnages, voire leurs échecs, et rétablit un ordre à la Nature. Il agit plutôt à la manière de la bouffonnerie de Don Quichotte, parvenant à embarquer le spectateur dans l'aventure, faisant de lui un compagnon de route. Il faudrait alors faire un portrait du spectateur de Valletti en Sancho Pança: en écuyer complice du théâtre mental du personnage. "L'histoire scandaleuse de Don Quichotte de la Manche et de son acolyte Sancho Pança», voilà décidément un juste paradigme du théâtre de Valletti. Le spectateur qui accompagne l'épopée de Don

\footnotetext{
8. Ibid.

9. Le jour se lève, Léopold! suivi de Souvenirs assassins, op. cit., p. Ізг.

Io. Tout est vécu, tentative d'entretien biographique avec Claude Guerre, Besançon, Les Solitaires intempestifs, 2004, p. 40.
} 
Quichotte est invité à regarder le monde à travers l'affabulation erratique et jubilatoire du personnage, et non pas à le juger ni à le sanctionner par le rire. Dans ce jeu entre Don Quichotte et Sancho Pança s'établit un accord tacite en vertu duquel le public accède à la scène mentale du personnage et se met à regarder le monde à travers sa vision et ses bizarreries.

\section{Un impressionnisme sans fin}

Ce que Sancho Pança découvre, c'est que l'aventure mythomane et chaotique des personnages de Valletti prend toujours pour point de départ l'ici-maintenant de la scène et le concret du monde. Il n'y aurait pas d'aventure chevaleresque pour Don Quichotte s'il ne rencontrait pas des moulins à vent bien réels, véritables appuis pour son épopée intérieure; pas non plus d'aventure théâtrale pour les héros de Valletti s'ils n'avaient pas à leur disposition la matérialité du plateau de théâtre et la présence effective du public. Car c'est bien de là que tout part: il faut d'abord le théâtre réel pour que le théâtre imaginaire puisse s'inventer. Le théâtre de Valletti se construit à partir d'un monde bien palpable, qui va être en quelque sorte absorbé par l'imaginaire du personnage pour devenir un théâtre complètement mental. Si l'on peut parler d' «impressionnisme» à propos de l'écriture de Valletti, ce n'est pas seulement en vertu de la composition éclatée, par petites touches, de l'œuvre ("C'est de l'impressionnisme littéraire", déclare le narrateur de l'Introduction ${ }^{\mathrm{II}}$ ); c'est aussi et peut-être surtout parce que toute parole y est le fruit d'une «impression», d'une marque imprimée par le réel sur la psychè ou l'imaginaire. Dans Plus d'histoires, l'auteur nous livre le mode de cette fabrique de l'impression en nous retraçant le chemin qui va du monde vers le moi; à propos des liasses de papiers qu'il porte sur lui, le personnage déclare:

Je me demande parfois si à force de les garder sur moi comme ça, eh bien ça ne coule pas le long de mes jambes et puis ça doit entrer par les pores, les pores de ma peau, comme les bateaux, ici en bas, ce sont des expressions, et puis ainsi s'immisçant sous l'épiderme, et puis le derme, dans le sang, à l'aide des vaisseaux remonte au cœur qui le distribue au cerveau et hop! je le retrouve sur les quelconques feuillets d'une liasse oubliée. ${ }^{\mathrm{I2}}$

Cette incroyable absorption du monde, qui est ici présentée de façon tout à fait physiologique, rend les personnages de Valletti complètement ouverts, presque béants parfois, tant ils ont absorbé de choses hétéroclites. L'être vallettien est fondamentalement habité par le monde; il en devient même squatté dans le cas de Souvenirs assassins. Et c'est dans cet effet d'ouverture

II. Introduction..., op. cit., p. 22.

I2. Plus d'histoires..., op. cit., p. 36. 
maximale que se fabrique le théâtre: tout du réel est susceptible de devenir matière à jouer, matériau pour l'aventure de la parole, rappelant le jeu des enfants qui se racontent les plus fabuleuses histoires à partir de bouts de ficelles. C'est aussi bien sûr comme cela que chemine Don Quichotte, qui s'empare de tous les objets du monde rencontrés sur la route pour se créer son aventure de chevalerie intérieure.

Cet impressionnisme est aussi un mouvement d'abstraction, puisque désormais, dans l'espace mental, le réel peut jouxter l'imaginaire, la fiction, la mythomanie sans que l'on ne puisse plus discerner la part des uns et des autres. Une fois reflué dans le théâtre mental, le réel se voit frappé du plus grand des soupçons: ce qui est dit existe-t-il? D'où est-ce que ça part? Et où est-ce que ça va? Par exemple, au début de Plus d'histoires, tout part de la réalité d'un théâtre en construction, à proximité du port et des bateaux. Mais très vite il apparaît que ce réel n'est plus si certain que cela:

Hier il y avait un peu plus de vent. C'était du vent qui venait de là! Aujourd'hui on dirait qu'il a changé un peu, il viendrait plus de là, on dirait! J'en mettrais pas ma main à couper, mais, c'est comme les impressions, on en jurerait pas, mais enfin, un peu comme hier mais plus de ce côté-là. ${ }^{13}$

Dans le duo intitulé L'Assassinat de John Fitzgerald Kennedy raconté à Aristote Onassis par Jacqueline Kennedy, Valletti met en scène une Jacqueline Kennedy ressassant sans fin les «impressions» laissées en elle par le moment de l'assassinat de son mari ; si le personnage rêve de remettre en ordre l'histoire - pour pouvoir l'oublier-, cette histoire ne cesse de lui échapper et de se déconstruire, devenant presque irréelle. La scène mentale n'est sûrement pas le lieu d'un tri ni d'une remise en ordre des impressions; c'est au contraire ce que Valletti appelle, dans la présentation de Si vous êtes des hommes!, un «cyclotron mental ${ }^{14} »$. Si toute parole jaillit à partir du concret de la scène, si la pensée côtoie très fortement le réel, pourtant le reflux du monde dans l'espace mental travaille ce même réel pour en faire un motif imaginaire, aussi léger qu'une particule dans le cyclotron. C'est sans doute la raison pour laquelle lire Valletti est une «entreprise désespérée», pour paraphraser Mallarmé, parce qu'on ne parvient pas, sur le papier, à bien discerner ce chemin qui va du concret à l'imaginaire, les lieux d'appuis réels qui vont mettre en mouvement le jeu des impressions dans la tête du personnage. Mais n'est-ce pas ce jeu du doute qui sur le plateau fait précisément théâtre? La force de cette dramaturgie tient à cette tension entre l'extrême concret de la parole, l'extrême présence du personnage captif à tout ce qui advient dans l'aventure, et l'extrême abstraction qui frappe le réel parce que ce dernier n'est plus qu'une impression sur la scène mentale. Ainsi, les

13. Ibid., p. 34 .

I4. Avant-propos de Si vous êtes des hommes!, op. cit., p. 9. 
épopées de Valletti sont toujours marquées du sceau de l'absence, de l'ailleurs. On ne sait si les histoires ont vraiment eu lieu ou si elles sont les produits de l'imaginaire débridé du personnage, d'un grand rêve impressionniste. Il n'est pas innocent que ce soit souvent entre la veille et le sommeil, entre le diurne et le nocturne, à la tombée de la nuit ou au lever du jour, que se situent les pièces de Valletti. "Ce spectacle est un passage entre la réalité de la ville et la fiction du théâtre, il est conseillé de le jouer à la tombée de la nuit ou bien au

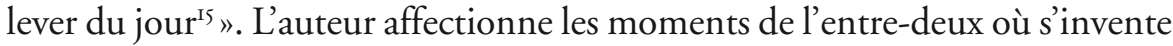
un théâtre de l'incertitude.

\section{De la folie baroque}

Cette tension entre réel et imaginaire ne recouvre jamais une opposition entre vérité et mensonge. L'imaginaire chez Valletti a une force de vérité aussi (voire plus) importante que le réel. À la fin de Saint Elvis, le personnage mythomane qui se prend pour le King est rappelé au réel mais résiste de toutes ses forces: à la vérité qu'on lui propose — il n'est pas Elvis Presley — il oppose sa sincérité absolue: «mais c'est ma vie ${ }^{16}$ !» s'exclame-t-il. Cette surévaluation de l'impressionnisme et de la mythomanie donne une dimension de folie aux personnages; elle les apparente encore une fois à Don Quichotte, pour qui l'aventure imaginaire est parfaitement vraie. Mais l'histoire d'un fou n'aurait rien d'intéressant si elle ne venait pas nous parler de nous tous, et si la folie ne pouvait pas devenir, à un moment, un paradigme pour regarder le monde autrement, en sortant des habitudes de la pensée et de la représentation. Comme le dit Claude Guerre dans son dialogue avec Valletti : "Et comment nous écarquiller mieux qu'en nous mentant pour nous dire la vérité, la vérité qui est un mensonge ${ }^{\mathrm{I7}}$ ?" Si le monde ne peut jamais être autre chose que sa représentation dans notre univers mental, ce qui nous apparaît comme des délires imaginaires peut recéler une extraordinaire poésie, voire un véritable bon sens. Les textes jouent ainsi d'un plaisir de la logique qui nous entraîne dans des endroits certes inattendus mais sûrement pas si fous que cela. À propos de la construction du théâtre, dans Plus d'histoires, le personnage explique:

Après on a fait des réunions, cinquante-huit réunions, pour savoir qu'est-ce qu'il $\mathrm{y}$ aurait dans l'endroit où on allait construire pour les représentations futures. Alors moi je me suis levé à un moment et j'ai dit: «Mais justement on ne peut pas savoir ce qu'il y aura dans cet endroit puisque c'est pour les représentations futures, or par principe le futur, on est pas des astrolabes! On ne peut pas le savoir le futur. $"{ }^{18}$

15. Avant-propos de Plus d'histoires..., op. cit., p. 33.

16. Saint Elvis, suivi de Carton plein, Paris, Christian Bourgois, 1990.

17. Tout est vécu..., op. cit., p. II.

18. Plus d'histoires..., op. cit., p. 54. 
Valletti aime avoir recours aux outils grammaticaux du raisonnement, de la déduction (donc, alors, voilà, parce que...), dans des démonstrations aussi folles que justes. À partir du moment où il entre dans la logique de la scène mentale, le spectateur est amené à donner raison au raisonnement distordu ou inattendu. Il ne réhabilite pas seulement Don Quichotte, il le fait triompher. Cette logique carnavalesque de fête des fous qui apportent la parole de raison fait songer bien sûr à l'univers théâtral de Shakespeare, et plus particulièrement à Yorrick, le fou d'Hamlet, que Valletti joua justement à Grenoble, dans le Hamlet mis en scène par Daniel Mesguich, et dont il est question dans le Sixième Solo. Les fous chez Shakespeare ont pour fonction de remettre le monde à l'endroit quand il est lui-même devenu fou et qu'il court au désastre. Voilà sans doute pourquoi Valletti parle dans Souvenirs assassins de «fou normal»: "J'étais fou. Mais comme j'étais un enfant on pensait que c'était normal»; "Fou comme on peut dire que le vent est fou. Tout simplement parce que personne ne sait où il va $»^{19}$. La métaphore du vent revient souvent dans l'écriture de Valletti pour désigner la folie du mouvement, folie qui n'est autre qu'une forme de liberté et un déconditionnement de la pensée. Le dramaturge fait un plaidoyer pour ce droit à la folie qu'on ne concède d'ordinaire qu'au vent, et que le personnage s'attribue sous le regard témoin et complice du spectateur. Car ce vent, c'est aussi la gratuité absolue de la poésie: «Et moi, bon à rien, incapable, la honte de ma famille de ne rien vendre d'autre que des riens, que ma voix, du vent, qu'est-ce que je vends? Rien, du vent, rien, que dalle ${ }^{20}$ !». Mais dans ce rien il y a tout, tout l'imaginaire. Les fous de Shakespeare ont souvent pour fonction de rappeler que l'homme est mortel et que toute propriété, toute gloire, n'est finalement que vanité... Ce motif baroque des vanités, des illusions qui masquent à l'homme la vérité de sa condition, revient dans la parole de Valletti pour raconter ce grand écart entre la prolifération des aventures et le rien, le vent qui les sous-tend. Si la scène mentale est gonflée comme un ballon de baudruche, elle menace à tout moment d'exploser, de crever et de témoigner de son vide — ou plutôt de sa plus grande humilité: «La devise du théâtre pourrait donc bien être: Vends du vent ${ }^{21}$ !» La fin de Plus d'histoires raconte cette tension entre le plein et le vide, entre le plus grand rêve et la conscience de l'humilité:

À ce moment précis où l'acteur qui joue prend conscience d'être en train de rater quelque chose, quelque chose qui se passerait en dehors et qui, s'il le voyait, rendrait dérisoire l'exact moment qu'il est en train de vivre. C'est pour ce risque

19. Souvenirs assassins, op. cit., p. II6-II7.

20. Just Hamlet, dans Cinq Duos, op. cit., p. 6I.

2I. Tout est vécu, op. cit., p. 31. 
insensé d'être dérisoire qu’on construit un lieu spécial qu’on appelle dans certains livres un Théâtre. ${ }^{22}$

Il y a un moment de bascule inattendu lorsque le fou rêveur reconnaît que son rêve n'existe pas, qu'il relève de l'utopie: "Ça existe! Bien sûr! bien sûr! Mais oui, je sais, ça n'existe pas! C'est de l'utopie, bien sûr! Mais on peut rêver! Alors oui, bien sûr ${ }^{23}$ !» L'étonnante lucidité des personnages de Valletti, dont nous avons ici un témoignage superbe, les fait ainsi échapper en fin de parcours à la figure de Quichotte — qui, lui, ne sort jamais de son rêve, qui n'en reconnaît jamais le caractère chimérique. Mais la reconnaissance d'une telle chimère chez Valletti n'empêche pas le personnage de continuer à rêver! Il y a de l'entêtement lucide chez ces mythomanes.

Derrière Quichotte surgit alors, peut-être, une ultime figure de la littérature baroque, celle d'Hamlet. Hamlet spectateur de sa propre vie, comme Valletti l'est de son propre théâtre mental, lui qui aime à rire de son rire; Hamlet qui a choisi la posture méditative et qui ne sait quel statut donner à ses fantômes, comme les mythomanes de Valletti partent à l'aventure sans fin de leurs impressions. Quelque chose de cette figure lucide et tragique se cache dans les interstices de l'armure de Don Quichotte, nous rappelant que la bouffonnerie ne peut atteindre sa dimension la plus haute que si elle contient en elle la conscience aiguë des ratés et de la vacuité de l'existence.

Et comme bien sûr c'est une illusion, que tout est aussi plat que tout à l'heure. Que je peux me retourner et regarder derrière moi sans avoir froid aux yeux. Que le vide est aussi bien devant que derrière. Et que Venise s'enfonce peu à peu et que jamais je ne pourrai empêcher sa mort. Que moi-même je suis en train de me décomposer à petit feu. ${ }^{24}$

22. Plus d'histoires..., op. cit., p. 64.

23. Ibid., p. 63.

24. Euf de lynch, dans Cinq Duos, op. cit., p. 88. 